\title{
Artificial organisms as tools for the development of psychological theory: Tolman's lesson
}

\author{
Orazio Miglino • Onofrio Gigliotta • \\ Maurizio Cardaci · Michela Ponticorvo
}

Received: 29 June 2007/ Accepted: 4 July 2007 / Published online: 31 July 2007

(C) Marta Olivetti Belardinelli and Springer-Verlag 2007

\begin{abstract}
In the 1930s and 1940s, Edward Tolman developed a psychological theory of spatial orientation in rats and humans. He expressed his theory as an automaton (the "schematic sowbug") or what today we would call an "artificial organism." With the technology of the day, he could not implement his model. Nonetheless, he used it to develop empirical predictions which tested with animals in the laboratory. This way of proceeding was in line with scientific practice dating back to Galileo. The way psychologists use artificial organisms in their work today breaks with this tradition. Modern "artificial organisms" are constructed a posteriori, working from experimental or ethological observations. As a result, researchers can use them to confirm a theoretical model or to simulate its operation. But they make no contribution to the actual building of models. In this paper, we try to return to Tolman's original strategy: implementing his theory of "vicarious trial and error" in a simulated robot, forecasting the robot's behavior and conducting experiments that verify or falsify these predictions.
\end{abstract}

\section{O. Miglino}

Department of Relational Sciences,

University of Naples "Federico II", Naples, Italy

O. Miglino $\cdot$ O. Gigliotta

Institute of the Sciences and Technologies of Knowledge,

National Research Council, Rome, Italy

O. Gigliotta - M. Cardaci

Department of Psychology and Interdepartmental Centre

of Knowledge Technologies, University of Palermo,

Palermo, Italy

M. Ponticorvo $(\square)$

Department of Linguistics, University of Calabria,

Cosenza, Italy

e-mail: michela.ponticorvo@unina.it
Keywords Schematic sowbug - Cognitive modeling . Artificial organisms · Tolman's theory

\section{Introduction}

Edward Tolman (1886-1959) was a major innovator in psychological theory. His works were written in an easygoing style and often poked subtle fun at academic orthodoxy. He drew on many, very different disciplines, from psychoanalysis and zoology, to Gestalt psychology and engineering. But unfortunately, his extreme eclecticism has left no trace in modern psychology. Today most scholars consider him a historical curiosity. All they remember of his thinking is his concept of a "cognitive map" and his reevaluation of organisms' autonomous cognitive activity in contrast with the radical behaviorism of his day. In reality, Tolman achieved far more than this. Among other things, he used robots as a tool, to understand learning. In this attempt, he was seventy years ahead of his time. Rereading his books and papers, we find the conceptual roots of several strands of modern research: the "Animat Approach" (Meyer 1995), "Cognitive Robotics" (Clark and Grush 1999), "Bio-morphic Robotics" (Assad et al. 2001), "Situated Systems" (Johnston 2001), "Evolutionary Robotics" (Nolfi and Floreano 2000), "Epigenetic Robotics" (Balkenius et al. 2004) and "Behavior-based robotics" (Arkin 1998). Tolman's idea of using robotic models in experimental psychology is more than a mere historical curiosity: from his work, it is possible to extract many ideas relevant to epistemological thinking on the use of robots in psychology (Webb 2000, Parisi 2005), as well as valuable methodological suggestions for experiments.

This is what we have tried to do in this paper. We have returned to a project that Tolman left unfinished and in a 
sense attempted to complete it. We began with a paper from 1939 in which Tolman used an automaton-what we would now call a "mobile robot"- to discuss a specific problem in psychological theory. Given the technological limitations of the time, Tolman's "machine" was never built. It remained a fanciful curiosity suggested by an eclectic scientist. A few years ago, Endo (Endo and Arkin 2001) built a robot partly inspired by Tolman's ideas. Endo's goal, however, was to build a machine displaying adaptive behavior - an engineering objective. By contrast, our goals, in the work reported here, were psychological. What we attempted to do was analyze and if possible reproduce the various stages Tolman went through in the construction of his model. This attempt has led us to rethink the use of robots in the construction of psychological theories.

In the next section, we introduce Tolman's work and its relevance to the epistemological debate on the role of models in psychology. In Sect. "Conclusions", we set out the theoretical problem to which Tolman applies his methodology; in Sect. 4 we describe his automaton; Sect. 5 introduces our own implementation. In Sect. 6, we compare our results with Tolman's predictions. Working from these findings, Sect. 7 attempts to define a reference procedure for robotic modeling which is sufficiently general to apply both to human and to animal behavior.

\section{Tolman's methodology: artificial organisms as theories, experiments with biological organisms}

Tolman adhered to a program of research whose aim was to achieve parity between psychology and the so-called "hard sciences". In this program, organisms' behavior was to be explained exclusively through application of the Galilean scientific method. In other words, experiments were to be derived from theories, expressed in formal (mathematical) terms. At the time Tolman was writing, it was widely believed that a mathematical theory of human action was within reach-indeed the attempt to build such a theory inspired almost all North American research in psychology, as we can see for example in Hull's monumental work (Hull 1943). In reality, with the exception of a few microtheories, the only result of this great collective effort was a multiplicity of constructs expressed in pseudo-formal language, based on poorly-defined formulae, and "box and arrow" diagrams (see the left part of Fig. 1). Tolman too was apt to adopt this approach. However, in the years of his human and scientific maturity, he realized its inadequacy and the confusion to which it could lead. So it was that in 1939, at the age of 53, he published what, for the time, was a curious and visionary paper. In the paper, he suggested a revolutionary new way of conceiving and presenting psychological theory. Here and in later publications, expanding on his original ideas (Tolman 1941, 1951; Tolman and Minium 1942), he proposed to express psychological theories in the form of artifacts equipped with sensors and motors-what today we would automatons, or "robots". His motivation for this proposal was not speculative but entirely practical. Tolman's intention was to place his theory of vicarious trial and error (VTE) behavior (see below) on a new footing. To achieve this, he proposed an automaton that he called the Schematic Sowbug. As already observed, he was not able to construct a physical version of his machine, but this did not prevent him from formulating predictions about its behavior. These were based not on calculation, but entirely on qualitative reasoning. Nonetheless, they enabled him to design and carry out a series of experiments on real animals, intended to corroborate or refute his theory (see below). To see his machine working he would have had to live in our own days.

Tolman first formulated his theory of VTE in the 1920s (Tolman 1925). Figure 1 shows the different ways in which he described the theory (see the next section). The left panel uses lines and arrows to depict the relations between independent, intervening and dependent variables, showing all the elements that, in Tolman's view, are necessary to explain the phenomenon. The right panel shows these same relations, incorporated into the sensory-motor structure of an "artificial organism" - the schematic sowbug. In practice, what Tolman was proposing was a new way of presenting a psychological theory. In this new approach, he replaced the complications of traditional psychological models with a "schematic" description of an entire organism. This suggests that we can develop theory by designing/implementing physical machines and studying the way they interact with their environment-just as we study the behavior of a laboratory animal. Traditional theory-building identified and analyzed the variables underlying psychological phenomena. Tolman reconstructed his abstract model in a machine, with a body and a sensorimotor system, whose behavior could be compared with that of a physical organism. This methodological innovation, a forerunner of Braitenberg's Synthetic Psychology (Braitenberg 1984), allowed him to imagine the way his artificial animal would behave in the real world. In substance it gave him the chance to imagine his theory in action.

Vicarious trial and error

One of the main interests of "behaviorist" psychologists in the first half of the last century was the study (and explanation) of decision-making. Choosing between alternative 
Fig. 1 From "boxes and arrows" to an "embodied" model [from the original drawing by Tolman (1939)]
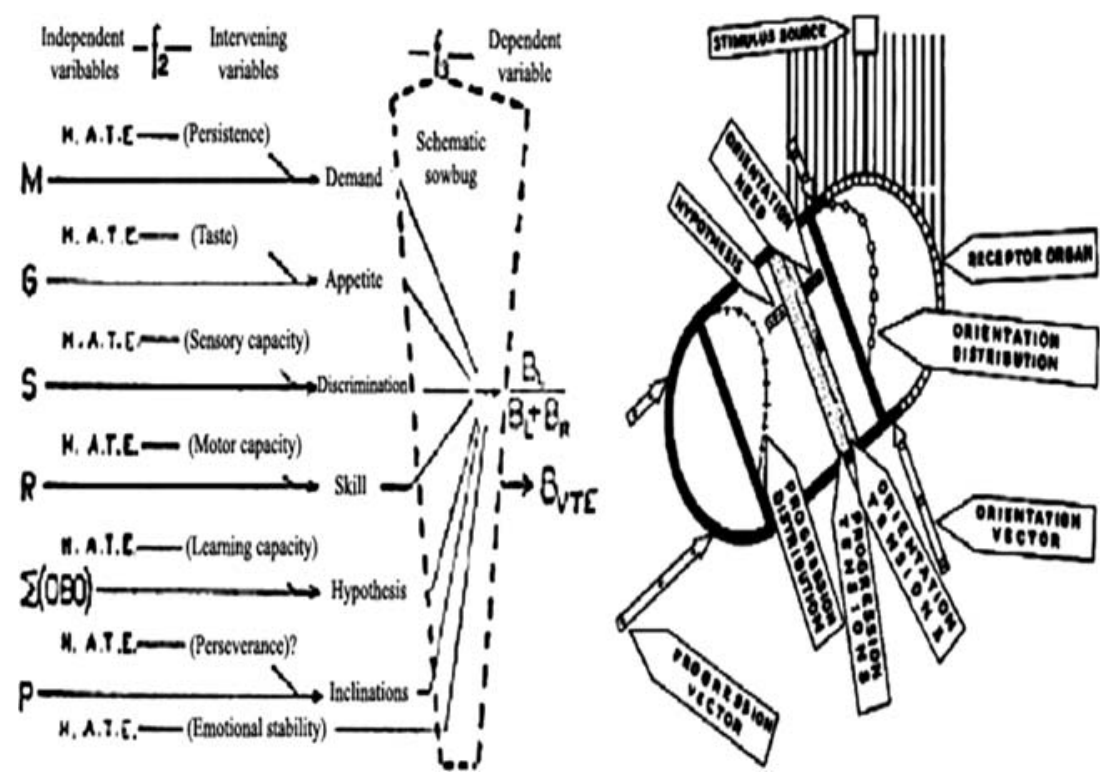

courses of action implies complex psychological activity, accompanied by very specific, sometimes paradoxical behaviors. One such behavioral paradox is so-called VTE. It has been repeatedly observed that, before making the correct choice between two options (two doors, two paths, two levers, etc.), many species of vertebrate (rats, monkeys, cats, pigeons) exhibit prolonged fixation first on one alternative, then on the other. This behavior has been interpreted as "mental" evaluation of the options at hand. In other words, for behaviorist psychologists, fixation on the alternatives replaces the physical act of choosing (touching, pressing, treading, etc.). Actual movement is inhibited. This is what has been called VTE.

Some of the behavioral sequences observed in organisms appear to be paradoxical (for a general review see Taylor and Reichlin 1951). In particular:

1. The number of correct responses over the trials (the learning curve) is directly correlated to the difference between the two stimuli.

2. VTEs are rare in the early stages of learning; thereafter they increase in frequency until the percentage of correct responses approaches $100 \%$, after which they become rapidly less common.

3. The number of VTEs is directly proportional to the difference between the two stimuli presented to the animal.

In other words, as organisms gradually learn to choose the right option (Observation 1), they hesitate more and more before reaching a decision (Observation 2: VTEs become more frequent). They hesitate most when the difference between the stimuli is greatest (Observation 3). Common sense suggests the opposite: the more organisms have learned-and the greater the difference between the alternatives - the faster we would expect them to decide. In his 1939 paper, Tolman proposed an explanation for these observations. He formulated his theory as a design for an "artificial organism"- the schematic sowbug.

An artificial organism as a theory

\section{The schematic sowbug as imagined by Tolman}

Tolman's design is entirely qualitative. The machine has approximately the shape of an egg (see the right side of Fig. 1) with a sensory apparatus (a set of light sensors) at one end and a motor apparatus at the other. In principle at least, Tolman' automaton receives visual stimuli from the external environment and can move in any direction it chooses. Its behavior is regulated by a series of rules. The totality of these rules constitutes a theory of VTE.

Tolman proposed a detailed classification of the rules governing the behavior of the automaton. This was profound yet entirely qualitative. It identified the relevant variables (for example: stimulus intensity, motor activation, the drive to act, etc.) in precise terms. However, the relations between them (the rules governing the sowbug's behavior) were expressed verbally, with no resort to formal language. This was not because Tolman was weak in mathematics-his first degree (from MIT) was in electrochemistry. Rather, the intricate interaction among the variables made a traditional mathematical formulation pointless - or in any case too obscure to be useful. In his model, Tolman had unwittingly introduced conceptsfeedback, automatic learning, information processing - that were difficult to handle with traditional mathematics. Proper definitions of these terms and tools to handle them 
Fig. 2 Vicarious trial and error in the Schematic Sowbug on tasks of varying difficulty: difficult (left), average difficulty (center) and easy (right). Adapted from Tolman (1939)

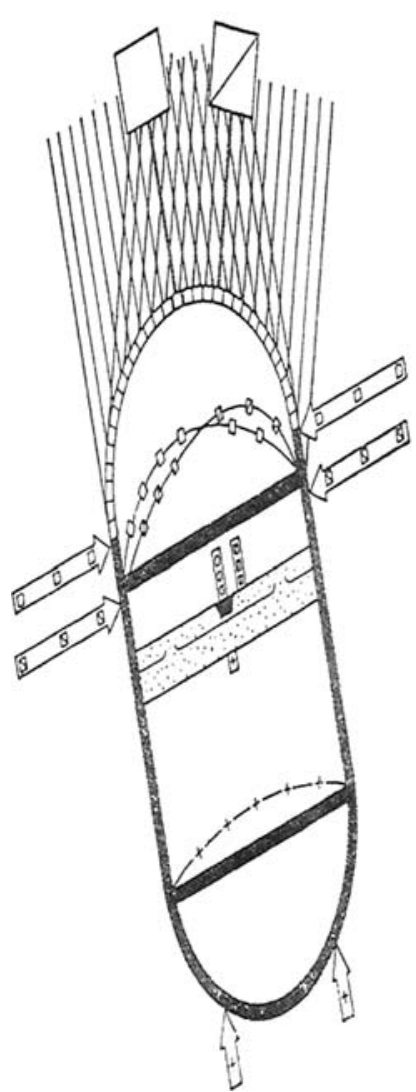

came years later with the birth of Cybernetics (Wiener 1948) and the invention of the computer. Only today has it become possible to transform Tolman's description into an algorithm which can be implemented on a computer (see later in this paper).

Tolman's quasi-algorithm is based on the theoretical approach of Lewin (Lewin 1936) and on studies of tropisms by Loeb (Loeb 1912) and Blum (Blum 1935). Lewin's Topological Psychology has influenced the work of a very large community of psychologists (especially social psychologists) and continues to be very influential even today. In topological psychology, an organism's mental organization is expressed as a topological map, locating the functions and information required for the organism's survival. In this view, individuals do not react automatically to stimuli, but filter them, mapping each stimulus onto a "mental" space. In line with this approach, Tolman, provided his automaton with a kind of map (its life space) representing the stimuli from its sense organs. Very different stimuli occupied distant points in life space; similar stimuli converged onto points which were close to each other. It was this structure of its life space-in modern terms the structure of its "cognitive representations" - that determined the Sowbug's behavior. Its behavioral repertoire consisted of two tropisms: orientation and approach. Given a stimulus, the Schematic

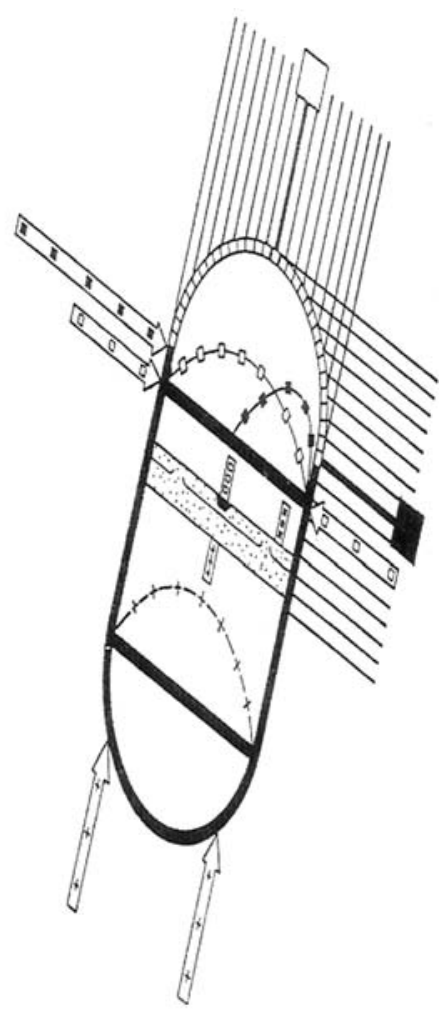

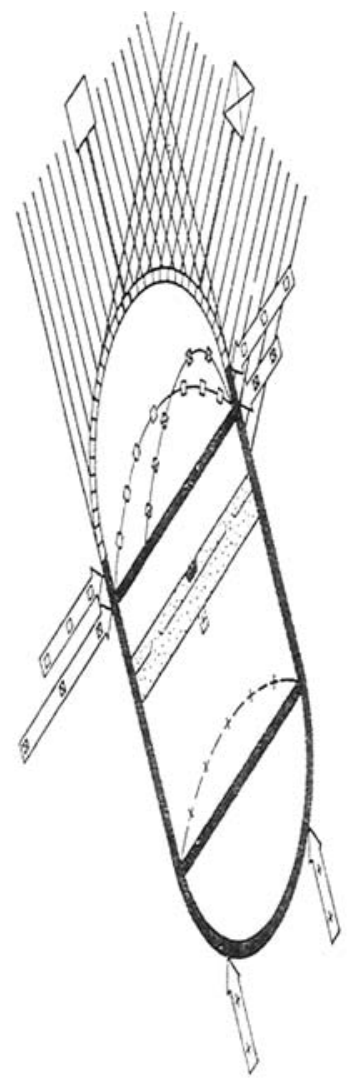

\section{}

Sowbug, would orient toward the stimulus (the orientation tropism) and move toward it (the approach tropism). In other words, the two tropisms act together but partly independently to determine the direction and the acceleration of the sowbug. Through repeated interactions with the environment (learning) the sowbug gradually acquires the approach tropism. The orientation tropism remains unchanged.

Tolman's description of the mechanism regulating the behavior of the Schematic Sowbug was long and not always straightforward or unambiguous. Using this description, he predicted the conditions under which the sowbug would display VTE (see Fig. 2). He then used these predictions to plan and implement animal experiments in the laboratory (see Sect. "From theoretical predictions to observable behavior: the design and implementation of experiments with biological organisms").

What he did not plan was a quantitative comparison between the predicted behavior of the sowbug and laboratory observations.

\section{Computer simulation of the schematic sowbug}

To quantify Tolman's theoretical predictions, we created a computer simulation of a physical structure consistent with 
Tolman's description of the schematic sowbug ${ }^{1}$ In our simulation, the sowbug is represented by a two-dimensional ellipsoidal structure composed of a $10 \mathrm{~cm} \times 5 \mathrm{~cm}$ rectangle lying between two semi-circles, each with a radius of $2.5 \mathrm{~cm}$. The simulated animal "lives" in a world populated by objects whose colors lie on a gray scale. Its sensory system, consisting of nine light sensors, lies at one pole of the ellipsoid. Each sensor has a receptive field of $180^{\circ}$. Sensor activation is inversely proportional to the distance from the source of stimulation and directly proportional to the level of gray of the stimulus (minimum in the case of a "white" object and maximum for a "black" object). This implies that the same sensor can produce different levels of activation even when it is stimulated from the same source. For example, if we place the Sowbug exactly opposite a stimulus of homogeneous color, its peripheral sensors, which are further away from the stimulus, will display a lower level of activation than sensors at the apex of the ellipsoid, which will function as a kind of "proto-fovea". According to Tolman, this property is a necessary condition for replicating the orienting behavior observed in VTE. He discussed the point at length, illustrating his argument in a diagram (see Fig. 3).

The Sowbug's motor apparatus consists of an orientation effector and a progress effector. The orientation effector allows the Sowbug to rotate around its axis by $\pm 45^{\circ}$; the progress effector has two states, 0 e 1 : when the effector is in state 0 the Sowbug does not move; when it is in state 1 (when the drive to approach exceeds the choice threshold), it jumps $10 \mathrm{~cm}$. in the direction of the sensory apparatus.

The experimental setting consists of a $50 \mathrm{~cm} \times 50 \mathrm{~cm}$ arena. At the beginning of each "training" session, the Sowbug is placed in the center of the arena, facing two rectangular objects of different colors, which are $14 \mathrm{~cm}$ apart (see Fig. 4). The experimenter assigns one object as "correct". The task for the Sowbug is to learn to choose this object (i.e., to move until it reaches the object).

The Sowbug's behavior is decided by a control system, whose main functions are shown in Fig. 5 and discussed below. Appendix 1 provides a formal description of the control system.

The stimuli (the two rectangular objects) activate the Sowbug's sensory system (Activity 1 ). If this is the first time the Sowbug has been exposed to the stimuli, the algorithm assigns values to three internal variables that represent the Sowbug's initial "knowledge base" (Activity 2). These are:

- The difference in color between the two stimuli; this measure is expressed by a value ranging from 0 (no

\footnotetext{
1 The program and the source code is written in Java. It can be downloaded from http://www.laral.istc.cnr.it/gigliotta/onisco.htm.
}

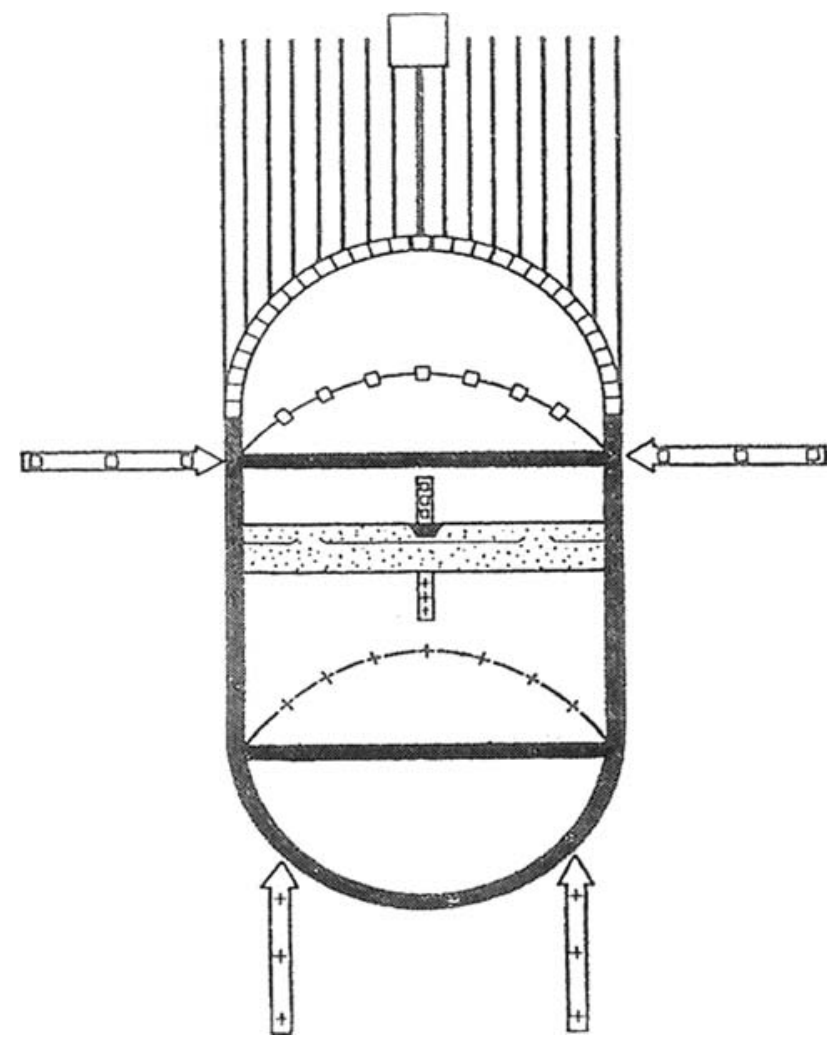

Fig. 3 The orienting reflex as a function of the structure of the Sowbug's sensory system. The Sowbug tends to maximize the total activation from its sensors. The optimal position for the stimulus is thus directly in front of the central sensors (adapted from Tolman 1939)

chromatic difference) to 1 (maximum chromatic difference: one object is white and the other is black) mapped in a probability space ranging from 0.5 to 1 (see appendix for values used in the simulation). In Tolman's terms this operation is equivalent to establishing the distance between the stimuli and placing them in the Sowbug's "life space" (see above).

- The level of "attraction" (Tolman calls this "Need") associated with each stimulus. This internal variable obliges the Sowbug to orient toward the stimulus with the higher level of attraction.

- The choice threshold associated with each stimulus: the more the Sowbug "looks" at a stimulus, the greater the drive to approach it. When the drive passes a threshold, the Sowbug approaches the stimulus.

The Sowbug activates its orientation effector depending on the sensory stimulation it receives and the values of its internal variables (Activity 3 ). If its new position leads it to focus on a single stimulus (the central sensors are excited by a single source of stimulation), its attraction to the stimulus diminishes drastically and its attraction to the other stimulus (which it is not focusing on) increases 
Fig. 4 The simulator, showing the results of a training session

Fig. 5 The sowbug control system

$\begin{array}{lll}\text { Onisco } 06 & \\ \text { File Simulation Settings } & \end{array}$
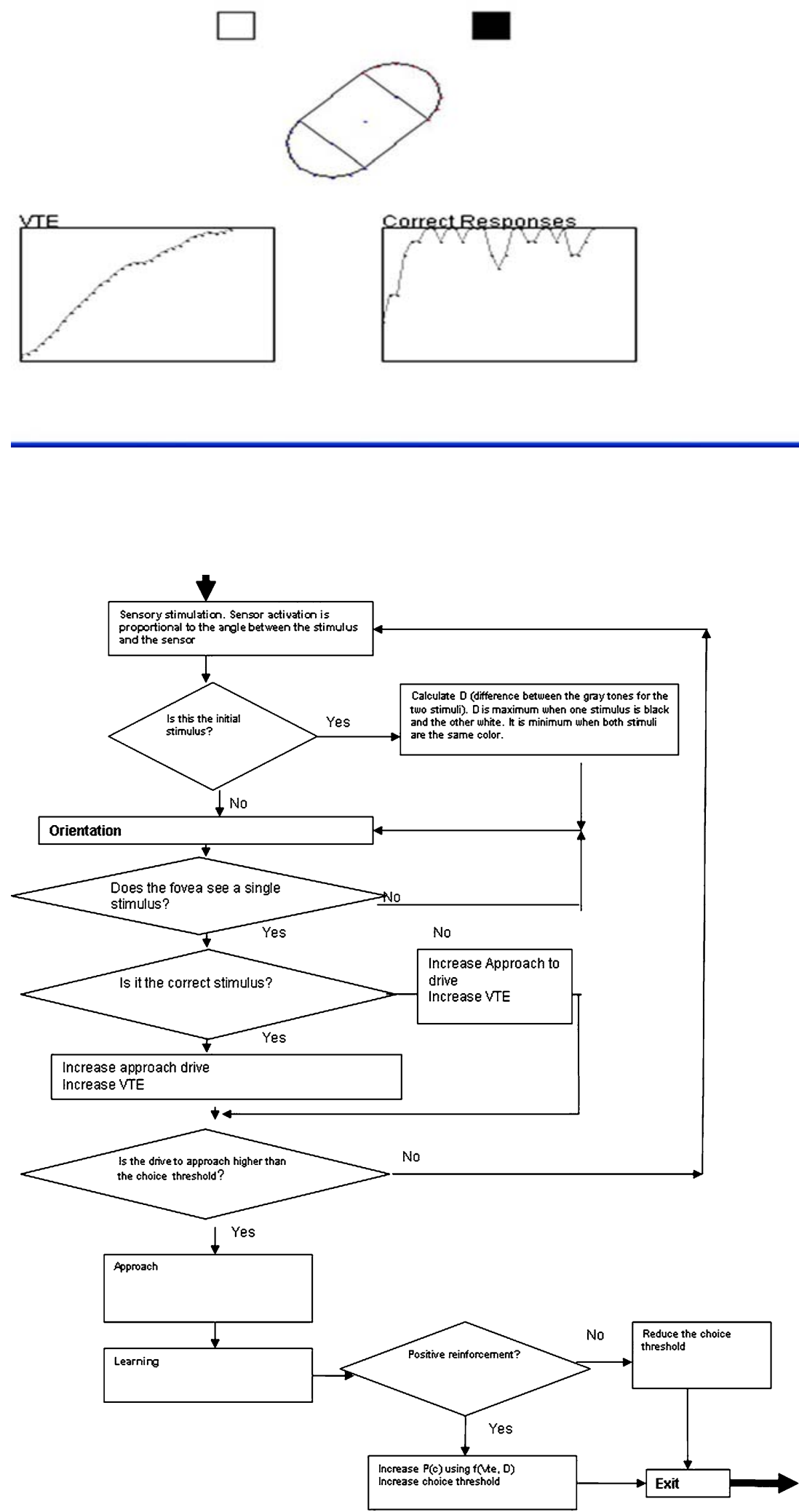
(Activity 4). This mechanism produces an oscillatory behavior in which the Sowbug focuses first on one stimulus then on the other.

Just as Tolman wrote in his paper, VTE plays a major role in the learning process. Whenever the Sowbug is focusing on a stimulus (VTE), the drive to approach will increase (Activity 4), according to Tolman for each VTE. When this drive passes a threshold, the Sowbug activates its approach effector (Activity 5). This behavior is interpreted as a concrete decision. At this point. a learning process (Activity 6) is triggered: if the stimulus chosen is the "correct" one, its choice threshold is increased as well as $\mathrm{P}(\mathrm{c})$ (i.e., the probability that the sowbug will make a correct response). Otherwise it is reduced. In other words, if it is easy to discriminate between the two stimuli, the choice threshold for the correct stimulus will increase during learning. If, on the other hand, the Sowbug chooses the wrong stimulus, the choice threshold will decrease. The whole mechanism is designed to ensure that the Sowbug becomes gradually more effective in choosing the right stimulus. In cases where there is little difference between the two stimuli, the Sowbug will make repeated errors.

In line with Tolman's reasoning, we conducted a series of experiments under three different conditions: high discrimination between stimuli (one white/one black), average discrimination (white/gray), low discrimination (white/ light gray). We observed that, for certain parameter (levels of attraction, choice thresholds, etc., see Appendix 1), the Sowbug perfectly reproduced the VTE behavior described in Sect. "Vicarious trial and error". Figures 6 and 7 describe the Sowbug's behavior in these experiments. The results match Tolman's qualitative predictions. Our simulations show he was also correct in quantitative terms.

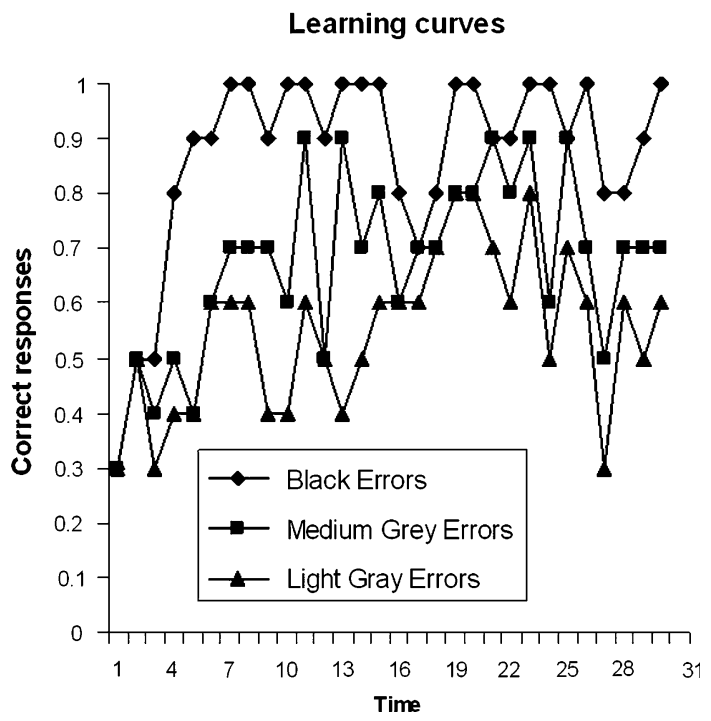

Fig. 6 Proportion of correct responses by session

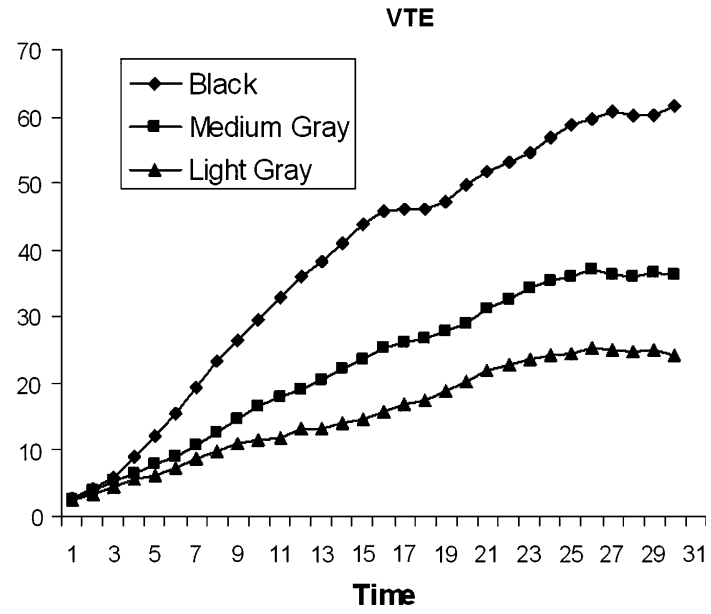

Fig. 7 Frequency of VTE by session. The Tolman model predicts a low number of VTEs in the initial phases of learning. VTEs are then expected to become more and more common until the animal has learned to reliably make the correct choice (the increased frequency of correct choices is shown in Fig. 6). The frequency of VTEs is directly proportional to the difference in color between the stimuli. VTEs are more common in discrimination between white and black stimuli than in whitelgray or whitellight gray discrimination tasks

From theoretical predictions to observable behavior: the design and implementation of experiments with biological organisms

After embodying his theory in the schematic Sowbug, Tolman went on to test its qualitative predictions in experiments with rats. Just like our own experiments with the simulated Sowbug, the experiments confirmed his theoretical predictions.

Given the importance of this result, it is worthwhile describing the main features of the experiment.

Tolman observed three groups of rats (mus norvegicus albinus) in an experimental setting originally devised by Lashley (1912), see Fig. 8). This setting was extremely similar to the setting in which Tolman had imagined his experiments with the Schematic Sowbug.

The rat was placed on a trestle in front of two colored doors. To escape, it had to choose which door to jump toward.

One group of rats was tested with doors (one white/one black) which it was easy for them to distinguish; another group was tested with doors (one white/one gray) which were fairly easy to distinguish; a third group was tested with doors that were difficult to distinguish (one white/one light gray). In all three cases, the door which led out of the maze was the white one. Therefore, it was this door that constituted the "correct" stimulus.

After a series of complicated experimental procedures, designed to investigate intervening variables (arrangement of the doors, standardization of the pre-training period, sex 


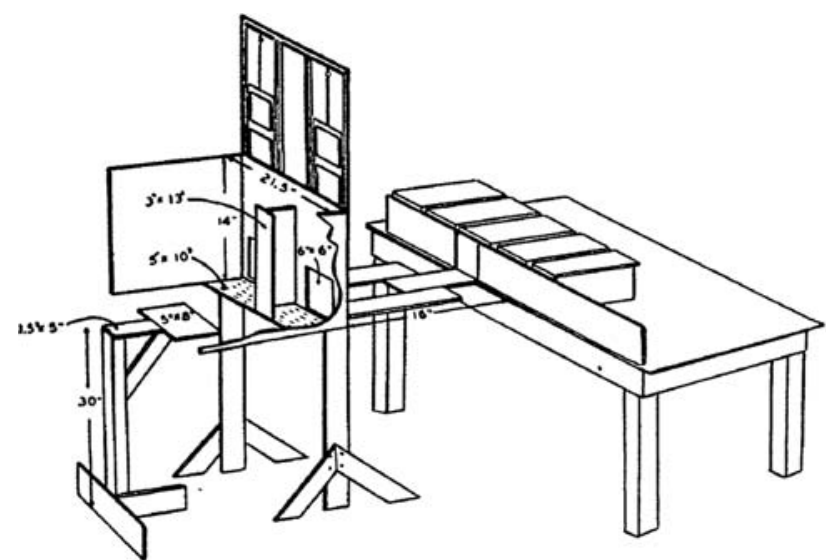

Fig. 8 The experimental apparatus used by Tolman in his experiment (adapted from Tolman 1939)

of rats, etc.), Tolman tested the rats on the experimental task. His experiments produced the learning curves shown in Figs. 9 and 10. Just as he had predicted, the rats produced the pattern of VTE described in Sect. "Vicarious trial and error".

\section{Conclusions}

In this paper, we have followed the intellectual journey that led Tolman to identify a certain behavioral phenomenon (VTE behavior), to formulate a theoretical explanation in the form of an Artificial Organism, to deduce empirical predictions and, finally, to devise theoretical predictions and to test them in a real laboratory experiment. Our own contribution is limited to the part of this project that

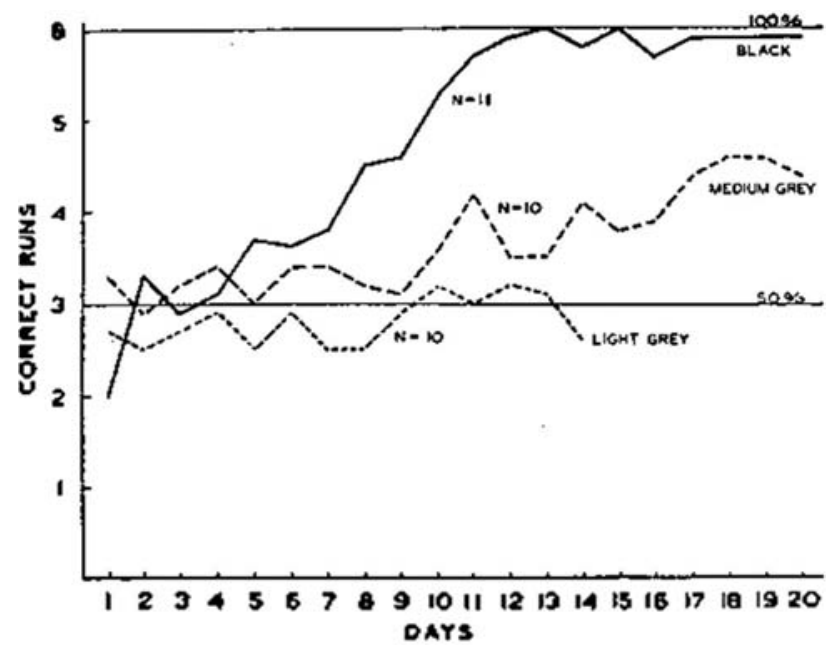

Fig. 9 Learning curves in relation to the average number of correct choices per day. The trend is compatible with the predictions reported in Fig. 6

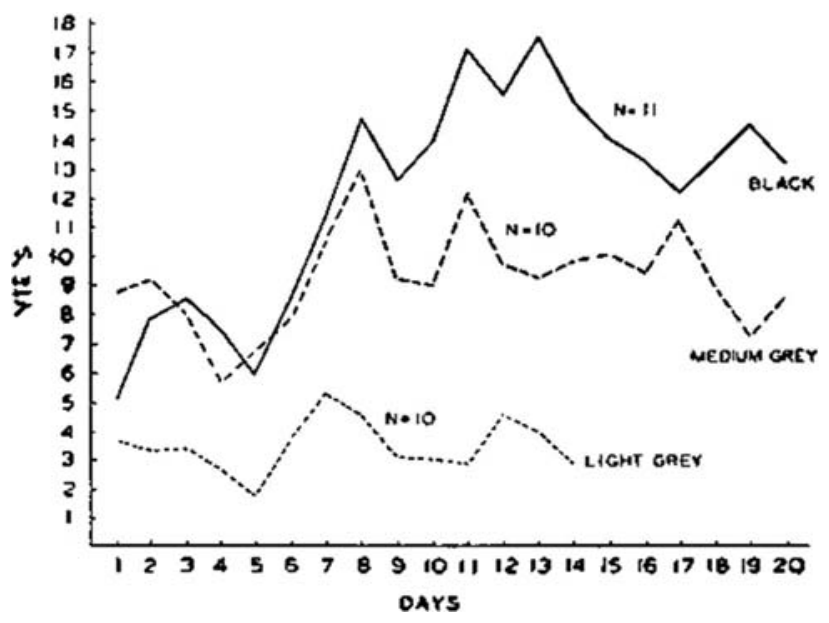

Fig. 10 Average number of VTE per day during the experiments. The trend is compatible with the predictions reported in Fig. 7

Tolman was unable to complete for lack of the necessary technology, namely the formalization of his theory in a computer program and the testing of its predictions. Our results suggest that, despite the criticism he suffered at the time, his approach could be extremely useful in producing scientific explanations of psychological phenomena.

Tolman was an innovator only in the way in which he expressed his theory. In all other respects he adopted the traditional Galilean method: he chose a phenomenon as his object of study, he hypothesized an explanation, he tested his predictions in a controlled laboratory experiment.

Today a large community of researchers involved in the study of the cognitive, neural and behavioral processes of living creatures accept Tolman's innovation, using "artificial organisms" to simulate a broad range of animal behavior. Several remarkable pieces of work in bioinspired robotics seem to adopt Tolman's strategy, even if not explicitly. For instance, Lund et al. (1998) used experiments with robot crickets not only to test data from biological experiments but to suggest future animal experiments.

However, most researchers in the field have taken a different path from Tolman's, using "artificial organisms" either as "ideal models" or as "data models". In the first case, they use them as a metaphor for phenomena that can be observed in nature. An example of this approach can be found in Nolfi (2005), an influential paper which shows how the behavior of Artificial Organisms (mobile robots) can be considered (and described) as a complex dynamic system. This method produces interesting heuristic insights. It does not however constitute a theory of any natural phenomenon.

An alternative approach is to use artificial organisms as a data model, precisely replicating experimental observations. In a recent paper, for example, Miglino and Walker 
(2004) tested mobile robots in an experimental setting originally used to study vertebrates' use of landmarks and geometrical cues in spatial cognition. Their findings showed a perfect match between the performance of "artificial organisms" and those of experimental animals. Using artificial organisms allowed them to observe their internal workings in ways which would not have been possible in a living organism. But it should be clear that this approach too was very different from Tolman's.

The key point is that Tolman's predictions preceded his animal experiments. In other words he used his (imagined) simulation to make empirical predictions. Only then did he design his experiments. Today we can go one step further. For the first time, technology allows us to construct "real" artificial organisms. The next challenge is to follow in Tolman's footsteps, transforming them from mere emulations of their biological equivalents into a method for developing scientific theories.

\section{Appendix 1}

Source code of the main java class used to simulate the sowbug.

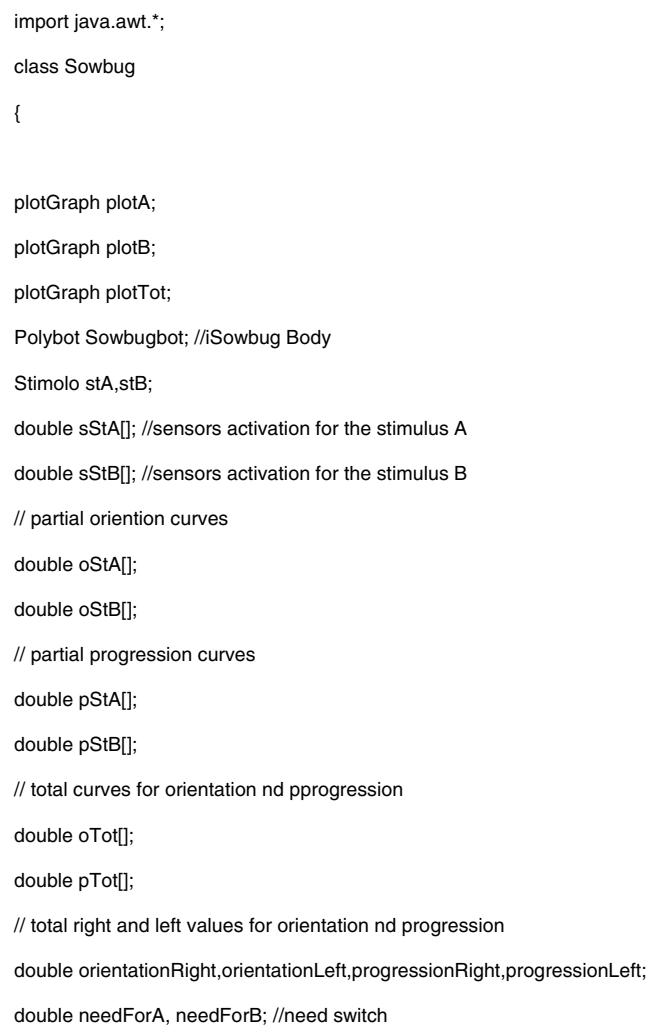


int errors;

// Number of incorrect responses

int currentStimulus; // means the status of the current stimulus: correct/incorrect

double correctOne;

Sowbug()

\{

//inizializzo vettori orientamento e progressione

sStA=new double[9];

sStB=new double[9];

oStA=new double[9];

oStB=new double[9];

pStA=new double[9];

pStB=new double[9];

oTot=new double[9];

pTot=new double[9];

//

plotA=new plotGraph(oStA,9,"Orient. Altro",120,270);

plotB=new plotGraph(oStB,9,"Orient. Bianco",10,270);

plotTot=new plotGraph(oTot,9,"Orient. Tot",230,270);

st $A=$ new Stimolo();

stB=new Stimolo();

initStimoli();

stB.setColoreStimolo(0);

stA.setColoreStimolo(3);

setStimoliPos $(90,100)$;

Sowbugbot=new Polybot(new Onishape());

Sowbugbot.setDirection(4);

resetExp();

needFor $\mathrm{A}=1$

needForB $=0$;

//Setting random numbers seed 
Sowbugbot.ur.random.setSeed(4);

//Initial values

chromaticDifference $=0.9 ; / /$ refers to the color of the Stimulus

$\mathrm{PC}=0.5$;

//Initial choice threshold

choiceThreshold=2

$k=100$;

\}

//Computing inputs:

public void getlnput()

\{

double distA, distB;

Punto2D rel=new Punto2D();

progressionRight $=0$

progressionLeft=0;

orientationRight=0;

orientationLeft $=0$;

for(int $i=0 ; i<9 ; i++)$

//Sensors Activation

rel.setX(Sowbugbot.coor_abs[i].getX()+Sowbugbot.coor_abs[i].getX()-

Sowbugbot.coor_abs[18].getX());

rel.setY(Sowbugbot.coor_abs[i].getY()+Sowbugbot.coor_abs[i].getY()-

Sowbugbot.coor_abs[18].getY());

$\operatorname{dist} \mathrm{A}=($ Sowbugbot.ur.distanzaAngolare(Sowbugbot.coor_abs[i],stA.pos,rel));

distB=(Sowbugbot.ur.distanzaAngolare(Sowbugbot.coor_abs[i],stB.pos,rel));

if (distA $>$ Math.PI/2) distA=Math.PI/2;

if (distB $>$ Math.PI/2) distB=Math.PI/2;

//sensors activation rely on the angular distance from the stimulus

$/ /$ more the sensor is near and more is the activation

sStA $[\mathrm{i}]=$ Math.cos $(\operatorname{dist} \mathrm{A})^{\star}$ Math.abs((stA.getColor()-stB.getColor())/90);//*(4-Math.abs $\left.((\mathrm{i}-4))\right) / 4$;

sStB[i]=Math.cos(distB)*Math.abs((stA.getColor()-stB.getColor())/90);//*(4-Math.abs((i-4)))/4; 


\author{
//computing orientation vector \\ oStA[i]=sStA[i];//*stA.getN(); \\ $p S t A[i]=s S t A[i]^{*} \operatorname{stA} \cdot g e t H()$; \\ oStB[i]=sStB[i];//*stB.getN(); \\ pStB $[i]=s S t B[i]^{*} \mathrm{stB} \cdot \operatorname{getH}()$; \\ oTot[i]=(oStA[i] ${ }^{*}$ needForA+oStB[i] ${ }^{*}$ needForB); \\ pTot[i]=(pStA[i]+pStB[i]);
}

for(int $i=0 ; i<4 ; i++)$

progressionLeft+=pTot[i];

orientationLeft+=oTot[i];

progressionRight+=pTot[i+5];

orientationRight $+=\mathrm{OTot}[\mathrm{i}+5]$;

\}

//Computing outputs:

public int getOutput()

\{

double $\mathrm{dO}, \mathrm{dP}, \mathrm{dT}$

$\mathrm{dO}=(\text { orientationRight-orientationLeft })^{*} 0.33333$;

$\mathrm{dP}=$ (progressionRight-progressionLeft);

$\mathrm{dT}=(\mathrm{dO})$;

Sowbugbot.turn(dT)

check();

if(noVte>choiceThreshold) 
\{

if $($ currentStimulus $==0$ )

\{

//choosed Stimulus is the correct one

//Increasing probability to give a correct response

$\mathrm{PC}+=(\text { chromaticDifference }-\mathrm{Pc})^{*}$ noVte $/\left(2^{*} \mathrm{k}\right)$;

//Increase choice threshold

choiceThreshold $+=$ chromaticDifference ${ }^{*}(\mathrm{k}-$ choiceThreshold $) / 200$;

if $($ currentStimulus==1)

\{

//choosed stimulus is the incorrect one

//decreasing choice threshold

choiceThreshold=choiceThreshold-chromaticDifference ${ }^{*}$ choiceThreshold/60;

incorrectResponses++;

\}

return -1 ;

\}

return 0

public void resetExp()

\{

resetPos();

resetTrial(); 
\}

public void resetTrial()

\{

double rnum

noVte=0;

errors $=0$;

stA.h $=0$;

stB.h $=0$;

resetPos();

rnum=Sowbugbot.ur.random.nextDouble();

//deciding what stimulus is correct in the current trial

if $($ rnum $<\mathrm{Pc})$

\{

currentStimulus $=0$;

needFor $\mathrm{A}=0$;

needForB $=1$;

\}

else

\{

currentStimulus $=1$;

needFor $\mathrm{A}=1$;

needForB=0;

\}

\}

public void paint(Graphics g)

\{

Sowbugbot.paint(g);

//drawing Sowbug 
g.setColor(Color.red);

for(int $i=0 ; i<9 ; i++)$

\{

g.fillOval(Sowbugbot.coor_abs[i].getIntX(),Sowbugbot.coor_abs[i].getIntY(),2,2);

\}

//drawing Stimuli

stA.paint(g);

stB.paint $(g)$;

public void resetPos()

//Resetting Sowbug initial position, needForAmely at the center of the areneedForA between two stimuli

Sowbugbot.reloadShape();

Sowbugbot.setPos(200,200);

Sowbugbot.turn(Math.PI);

public void initStimoli()

stA.setColor(30);

stA.h $=0$;

stB.setColor(0);

stB. $h=0$

public void setStimoliPos(double degrees, double radius)

double alfa; 
alfa $=$ degrees ${ }^{*}{ }^{*} \mathrm{Math} . \mathrm{PI} / 360$;

stA.pos.setXY(200+Math.cos(alfa/2)*radius,200-Math.sin(alfa/2)*radius);

stB.pos.setXY(200+Math.cos(Math.PI-alfa/2)* ${ }^{\star}$ radius,200-Math.sin(Math.PI-alfa/2)*radius);

\}

public void check()

\{

//checking whether the Sowbug is facing or not a stimuls

//if yes the need for that stimulus decrease increasing the need

//for the other stimulus

if (Math.abs(Sowbugbot.ur.distanzaAngolare(Sowbugbot.pos,stA.pos,Sowbugbot.coor_abs[4]))<0.01)

\{

needForA $=0$;

needForB=1;

noVte++;

\}

if (Math.abs(Sowbugbot.ur.distanzaAngolare(Sowbugbot.pos,stB.pos,Sowbugbot.coor_abs[4]))<0.01)

\{

needForA=1;

needForB=0;

noVte++;

\}

\section{References}

Arkin RC (1998) Behavior-based robotics. MIT Press, Cambridge Assad C, Mitra JH, Lewis A (2001) Introduction to the special issue on biomorphic robotics. Auton Robots 11:195-200

Balkenius C, Zlatev J, Kozima H, Dautenhahn K, Breazeal C (2004) Proceedings of the first international workshop on epigenetic robotics: modeling cognitive development in robotic systems. Lund University Cognitive Studies

Blum HF (1935) An analysis of oriented movements of animals in light fields. Cold Spring Harb Symp Quant Biol 3:210-223

Braitenberg V (1984) Vehicles: experiments in synthetic psychology. MIT Press, Cambridge
Clark A, Grush R (1999) Towards a cognitive robotics. Adaptive Behavior 7(1):5-16

Endo Y, Arkin R C (2001) Implementing Tolman's schematic Sowbug: behavior-based robotics in the 1930s. Paper presented at the IEEE international conference on robotics and automation (ICRA), Seoul

Hull CL (1943) Principles of behavior. Appleton, New York

Johnston RB (2001) Situated action, structuration and actor-network theory: an integrative perspective. Paper presented at the ECIS 2001, 9th European conference on information systems, Bled

Lashley KS (1912) Visual discrimination of size and form in the albino rat. J Anim Behav 2:310-331

Lewin K (1936) Principles of topological psychology. McGraw-Hill, New York 
Loeb J (1912) The mechanistic conception of life: biological essays. The University of Chicago Press, Chicago

Lund HH, Webb B, Hallam J (1998) Physical and temporal scaling considerations in a robot model of cricket calling song preference. Artif Life 4(1):95-107

Meyer JA (1995) The animat approach to cognitive science. In: Roitblat HL, Meyer JA (eds) Comparative approaches to cognitive science. The MIT PressBradford Books, Cambridge

Miglino O, Walker R (2004) An action-based mechanism for the interpretation of biometrical clues during navigation. Connection Science 16(4):267-281

Nolfi S (2005) Behaviour as a complex adaptive system. Paper presented at the European conference on complex systems (ECCS'05), Paris

Nolfi S, Floreano D (2000) Evolutionary robotics: the biology, intelligence, and technology of self-organizing machines. MIT Press/Bradford Books, Cambridge
Parisi D (2005) Robot come psicologia. In: Peruzzi A (ed.) Atti del convegno pianeta Galileo

Taylor JG, Reichlin B (1951) Vicarious trial and error. Psychol Rev 58(6):389-402

Tolman EC (1925) Purpose and cognition: the determiners of animal learning. Psychol Rev 32:285-308

Tolman EC (1939) Prediction of vicarious trial and error by means of the schematic sowbug. Psychol Rev 46:318-336

Tolman EC (1941) Discrimination vs. learning and the schematic sowbug. Psychol Rev 48:367-382

Tolman EC (1951) Behavior and psychological man. University of California Press, Berkeley

Tolman EC, Minium E (1942) VTE in rats: overlearning and difficulty of discrimination. J Comp Psychol 34:303

Webb B (2000) What does robotics offer animal behaviour? Anim Behav 60:545-558

Wiener N (1948) Cybernetics: or control and communication in the animal and the machine. MIT Press, Cambridge 